\title{
ON A SUBCLASS OF SQUARE INTEGRABLE MARTINGALES
}

\author{
DEAN ISAACSON
}

\begin{abstract}
Let $\mathscr{H}_{2}^{*}$ denote the class of continuous, nowhere constant, square integrable martingales, $M(t)=X\left(\langle M\rangle_{t}\right)$, for which $\langle M\rangle_{t}$ is a time change on the $\sigma$-fields generated by the Brownian motion $X(t)$. It is shown that if $M(t) \in \mathscr{M}_{2}^{*}$, then the family of $\sigma$ fields generated by $M(t)$ is a right continuous family. If $M(t) \in \mathscr{M}_{2}^{*}$ and if $\sigma\{M(s): s \leqq t\}=\sigma\{X(s): s \leqq t\}$ for some Brownian motion $X(t)$, then $M(t)=\int_{0}^{t} \Phi(s) d X(s)$ and $X(t)=\int_{0}^{t}(1 / \Phi(s)) d M(s)$ for some process $\Phi(s)$ with $\Phi(s) \neq 0$ a.e. $d t \times d P$.
\end{abstract}

1. Introduction. In this paper all processes will be defined on a probability space, $(\Omega, \mathscr{F}, P)$, and all $\sigma$-fields will be completed with respect to $P$. The class $\mathscr{M}_{2}$ of continuous, square integrable, nowhere constant, martingales with $M(0)=0$ is a frequently studied class with many nice properties. For example, it is well known that if $(M(t), \mathscr{G}(t))_{t \geqq 0} \in \mathscr{M}_{2}$, then $M(t)$ can be expressed as a time change on a Brownian motion [4]. That is, there exists a Brownian motion $(X(t), \mathscr{F}(t))$ such that $M(t)=X(A(t))$ where $A(t)=\langle M\rangle_{t}$ is the natural increasing process arising in the Doob decomposition of $M^{2}(t)$. In this paper we will concern ourselves with the additional question of whether or not $A(t)$ is a time change relative to $H(t)=\sigma\{X(s): s \leqq t\}$. The following example will show that not every process in $\mathscr{M}_{2}$ has this property. Let $(X(t), \mathscr{F}(t))_{t \geqq 0}$ be a Brownian motion with $\mathscr{F}(t)=\sigma\{X(s): s \leqq t\}$. Let $B$ be a subset of $\Omega$ that is independent of $\mathscr{F}(\infty)$ with $0<P(B)<1$. Then $(X(t), \sigma\{\mathscr{F}(t) \cup B\})_{t \geqq 0}$ is a Brownian motion. Let $A(t)$ be a time change defined by $A(t)=t$ if $\omega \in B$ and $A(t)=2 t$ if $\omega \in B^{c}$. Define $M(t)=X(A(t))$. Then $M(t) \in \mathscr{M}_{2}$ but there is no Brownian motion $Y(t)$ such that $A(t)$ is a time change on $\sigma\{Y(s): s \leqq t\}$. In particular $H(t)=\sigma\{Y(s): s \leqq t\}$ is a right continuous family with $H(0)$ a trivial $\sigma$-field [1, p. 42] and in order for $A(t)$ to be a stopping time on $H(t)$ one must have $B \in H(t)$ for all $t>0$. In the remainder of this paper we will denote by $\mathscr{M}_{2}^{*}$ the subset of $\mathscr{M}_{2}$ consisting of processes $M(t)$ for which $A(t)=\langle M\rangle_{t}$

Received by the editors September 6, 1971.

AMS 1970 subject classifications. Primary 60G45.

Key words and phrases. Continuous square integrable nowhere constant martingales, Brownian motion, time change, stochastic integral.

(c) American Mathematical Society 1972 
is a time change on a family of $\sigma$-fields generated by a Brownian motion $X(t)$ and $M(t)=X(A(t))$.

2. Processes generating a right continuous family of $\sigma$-fields. In many theorems involving martingales one encounters the assumption that the family of adapted $\sigma$-fields be right continuous (see for example [9]). On the other hand it is often assumed that the adapted family is the family generated by the process. The first question we consider is whether or not the family of $\sigma$-fields generated by a process is right continuous. The above example shows that processes in $\mathscr{M}_{2}$ do not necessarily generate a right continuous family, however, we will show that processs in $\mathscr{M}_{2}^{*}$ do. Before stating this theorem, we need two lemmas. The first lemma is known [2] but is included here for the convenience of the reader.

Lemma 1. Let $(H(t))_{t \geqq 0}$ and $(\mathscr{G}(t))_{t \geq 0}$ be families of $\sigma$-fields on $\Omega$. Let $A(t)$ he a time change on $\mathscr{G}(t)$ with $H(t)=\mathscr{G}(A(t))$ and let $B(t)$ be a time change on $H(t)$. If $A(t)$ and $B(t)$ have continuous paths, then $H(B(t))=$ $\mathscr{G}(A(B(t)))$.

Proof. Let $E \in H(B(t))$ so $E \cap[B(t)>\lambda] \cap[A(\lambda)>\alpha] \in \mathscr{G}(\alpha)$ for all $\alpha \geqq 0, \lambda \geqq 0$. Hence $E \cap[B(t)>\lambda] \cap\left[\lambda>A^{-1}(\alpha)\right] \in \mathscr{G}(\alpha)$ for all $\alpha \geqq 0, \lambda \geqq 0$, where $A^{-1}(\alpha)=\inf \{t: A(t)>\alpha\}$ and $A^{-1}(\alpha)=\infty$ if the set is empty. Now taking the union over all rational $\lambda$, one gets

$$
E \cap\left[A^{-1}(\alpha)<B(t)\right] \in \mathscr{G}(\alpha) \text { for all } \alpha \geqq 0 .
$$

Hence $E \cap[A(B(t))>\alpha] \in \mathscr{G}(\alpha)$ for all $\alpha \geqq 0$, so $H(B(t)) \subset \mathscr{G}(A(B(t)))$.

To show the inclusion the other way, assume $E \in \mathscr{G}(A(B(t)))$. Then $E \cap\left[B(t) \leqq A^{-1}(\alpha)\right] \in \mathscr{G}(\alpha)$ for all $\alpha \geqq 0$. Now $[B(t) \leqq \lambda] \in H(\lambda)=\mathscr{G}(A(\lambda))$ for all $\lambda \geqq 0$, so $[B(t) \leqq \lambda] \cap[A(\lambda) \leqq \alpha] \in \mathscr{G}(\alpha)$ for all $\lambda \geqq 0, \alpha \geqq 0$. Hence

$$
\begin{aligned}
E \cap\left[B(t) \leqq A^{-1}(\alpha)\right] \cap[B(t) \leqq \lambda] \cap\left[\lambda \leqq A^{-1}(\alpha)\right] \in \mathscr{G}(\alpha) \\
\text { for all } \alpha \geqq 0, \lambda \geqq 0,
\end{aligned}
$$

so

$$
E \cap[B(t) \leqq \lambda] \cap\left[\lambda \leqq A^{-1}(\alpha)\right] \in \mathscr{G}(\alpha) \text { for all } \alpha \geqq 0, \lambda \geqq 0
$$

and hence $E \in H(B(t))$.

Lemma 2. Let $X(t)$ be a Brownian motion process and let $\mathscr{F}(t)=$ $\sigma\{X(s): s \leqq t\}$. If $A(t)$ is a continuous and strictly increasing time change on $\mathscr{F}(t)$ with $A(0)=0$ and $E[A(t)]<\infty$ for all $t \geqq 0$, then $\mathscr{F}(A(t))=$ $\sigma\{X(A(s)): s \leqq t\}$.

Proof. For notational convenience let $M(t)=X(A(t))$ and $H(t)=$ $\sigma\{M(s): s \leqq t\}$. Note that $M_{t} \in \mathscr{M}_{2}^{*}$. Since both $H(t)$ and $\mathscr{F}(A(t))$ support $M(t)$ we have $H(t) \subset \mathscr{F}(A(t))$. Define $A^{-1}(t)$ as above. This process is by 
definition a time change on $\mathscr{F}(A(t))$ but it must be shown to be a time change on $H(t)$. Now

$$
\left[A^{-1}(t) \leqq \lambda\right]=[t \leqq A(\lambda)]
$$

and

$$
A(\hat{\lambda})=\lim \sum_{k=1}^{n}\left(M\left(t_{k}\right)-M\left(t_{k-1}\right)\right)^{2}
$$

where $0=t_{0}<t_{1}<\cdots<t_{n}=\lambda$, and the limit is a limit in probability as $n \rightarrow \infty$ and $\max _{k}\left|t_{k}-t_{k-1}\right| \rightarrow 0[8]$. Hence $[t \leqq A(\lambda)] \in H(\lambda)$ so $A^{-1}(t)$ is a time change on $(M(t), H(t))$. For now we will assume $\lim _{t \rightarrow \infty} A(t)=\infty$ a.s. so that $A\left[A^{-1}(t)\right]=t$ a.s. for all $t$. Under this assumption, $H\left(A^{-1}(t)\right)$ and $\mathscr{F}\left(A\left(A^{-1}(t)\right)\right)=\mathscr{F}(t)$ both support $X\left(A\left(A^{-1}(t)\right)\right)=X(t)$ and $H\left(A^{-1}(t)\right) \subset$ $\mathscr{F}(t)$. However, $\mathscr{F}(t)$ was constructed to be minimal for $X(t)$ so $H\left(A^{-1}(t)\right)=$ $\mathscr{F}(t)$ which implies $H(t)=\mathscr{F}(A(t))$. If one does not assume $\lim _{t \rightarrow \infty} A(t)=$ $\infty$ a.s., then define a new time change $A^{n}(t)$ as follows:

$$
\begin{aligned}
A^{n}(t) & =A(t) & & \text { for } t \leqq n, \\
& =A(t)+(t-n) & & \text { for } t>n .
\end{aligned}
$$

$A^{n}(t)$ is still a time change on $\mathscr{F}(t)$ with $\lim _{t \rightarrow \infty} A^{n}(t)=\infty$. By the first case we know $\mathscr{F}\left(A^{n}(t)\right)=\sigma\left\{X\left(A^{n}(s)\right): s \leqq t\right\}$. However, for $t \leqq n$ we have $\mathscr{F}\left(A^{n}(t)\right)=\mathscr{F}(A(t))$ and $\sigma\left\{X\left(A^{n}(s)\right): s \leqq t\right\}=\sigma\{X(A(s)): s \leqq t\}$. Therefore $\mathscr{F}(A(t))=\sigma\{X(A(s)): s \leqq t\}$ for all $t \geqq 0$.

THEOREM 1. If $M(t) \in \mathscr{M}_{2}^{*}$ then $H(t)=\sigma\{M(s): s \leqq t\}$ is a right continuous family.

Proof. Let $M(t)=X(A(t))$ where $(X(t), \mathscr{F}(t))$ is a Brownian motion with $\mathscr{F}(t)=\sigma\{X(s): s \leqq t\}$. It is known that $\mathscr{F}(t)$ is a right continuous family [1]. Hence one can easily show that $\mathscr{F}(A(t))$ is a right continuous family [9, p. 67]. The theorem now reduces to showing $H(t)=\sigma\{M(s): s \leqq t\}=$ $\mathscr{F}(A(t))$. Since for $M(t) \in \mathscr{M}_{2}$ we have $A(t)$ is of the type in Lemma 2 , the result follows. In view of the above discussion one might conjecture that for $M(t) \in \mathscr{M}_{2}$ if $\sigma\{M(s): s \leqq t\}$ is a right continuous family, then $M(t) \in$ $\mathscr{M}_{2}^{*}$. This is an open question as far as the author knows.

3. Martingales as stochastic integrals with nonzero integrands. In order to motivate the next theorem we review some known results. Let $(M(t)$, $\mathscr{G}(t)$ ) belong to $\mathscr{M}_{2}$. It is known that the following are equivalent [5].

(i) $\langle M\rangle_{t}$ is absolutely continuous with respect to Lebesgue measure and $d\langle M\rangle_{t} \mid d t>0$ a.e. $d t \times d P$.

(ii) $M(t)$ can be expressed as $M(t)=\int_{0}^{t} \Phi(s) d X(s)$ where $\Phi(s) \neq 0$ a.e. $d t \times d P$ and $(X(t), \mathscr{F}(t))$ is a Brownian motion process. In this case $\Phi(s)=\left(d\langle M\rangle_{s} / d s\right)^{1 / 2}$ a.e. $d t \times d P$. 
If one only has $d\langle M\rangle_{t} / d t \geqq 0$ a.s. then by adjoining a Brownian motion to $(M(t), \mathscr{G}(t))$ we get the following which are equivalent [3, p. 449].

(i') $\langle M\rangle_{t}$ is absolutely continuous with respect to Lebesgue measure.

(ii') $M(t)$ can be expressed as $M(t)=\int_{0}^{t} \Phi(s) d X(s)$ where $(X(t), \mathscr{F}(t))$ is a Brownian motion process and again $\Phi(s)=\left(d\langle M\rangle_{s} / d s\right)^{1 / 2}$.

The following condition is known to imply (ii') [8, p. 135].

(iii') $M(t)$ is a martingale on $[0, \infty)$, that is adapted to the family of $\sigma$ fields generated by the Brownian motion process $X(t)$.

The question we now consider is, what analogous condition would imply (ii) or (i)?

THEOREM 2. Let $(M(t), \mathscr{G}(t))_{t \geqq 0}$ belong to $\mathscr{M}_{2}^{*}$ and let $(X(t), \mathscr{G}(t))_{t \geqq 0}$ be a Brownian motion with $\mathscr{G}(t)=\sigma\{X(s): s \leqq t\}$. Then $M(t)=\int_{0}^{t} \Phi(s) d X(s)$ with $\Phi \neq 0$ a.e. $d t \times d P$ and $\Phi(t)$ adapted to $\sigma\{X(s): s \leqq t\}$ and $\sigma\{M(s): s \leqq t\}$ if and only if $\mathscr{G}(t)=\sigma\{M(s): s \leqq t\}$.

Proof. Assume $M(t)=\int_{0}^{t} \Phi(s) d X(s)$ with $\Phi(t)$ adapted to $\sigma\{X(s): s \leqq t\}$. Then $M(t)$ is a martingale with respect to $\sigma\{X(s): s \leqq t\}$ so $\sigma\{M(s): s \leqq t\} \subset$ $\sigma\{X(s): s \leqq t\}$. Also since $\Phi(s) \neq 0$ one can write $X(t)=\int_{0}^{t}(1 / \Phi(s)) d M(s)$ with $1 / \Phi(s)$ adapted to $\sigma\{M(s): s \leqq t\}$ so $\sigma\{X(s): s \leqq t\} \subset \sigma\{M(s): s \leqq t\}$.

Conversely assume $\sigma\{X(s): s \leqq t\}=\sigma\{M(s): s \leqq t\}$. Let $A(t)=\langle M\rangle_{t}$ and let $A^{-1}(t)=\inf \{s: A(s)>t\}$ as before.

Case 1. Assume $E\left[A^{-1}(t)\right]<\infty$ for every $t$, which implies $A\left(A^{-1}(t)\right)=t$ a.s. From the hypothesis we get $\left[8\right.$, p. 135] $M(t)=\int_{0}^{t} \Phi(s) d X(s)$ with $\Phi(t)$ adapted to $\sigma\{X(s): s \leqq t\}$ and also $A(t)=\int_{0}^{t} \Phi^{2}(s) d s$. Now $A^{-1}(t)$ is a time change on $\mathscr{G}(t)$ so consider $\left(M\left(A^{-1}(t)\right), \mathscr{G}\left(A^{-1}(t)\right)\right)$ and $\left(X\left(A^{-1}(t)\right)\right.$, $\left.\mathscr{G}\left(A^{-1}(t)\right)\right)$. Since $E\left[A^{-1}(t)\right]<\infty$ we have $X\left(A^{-1}(t)\right) \in \mathscr{M}_{2}^{*}$ [2] and $\mathscr{G}\left(A^{-1}(t)\right)=\sigma\left\{X\left(A^{-1}(s)\right): s \leqq t\right\}$ by Lemma 2 . From the proof of Theorem 1 we know $\mathscr{G}(t)=\mathscr{F}(A(t))$ where $\mathscr{F}(t)$ is a family of $\sigma$-fields generated by a Brownian motion $Y(t)$. Hence $X\left(A^{-1}(t)\right)$ is adapted to $\mathscr{G}\left(A^{-1}(t)\right)=\mathscr{F}(t)$ so $X\left(A^{-1}(t)\right)=\int_{0}^{t} \psi(s) d Y(s)$. This implies $A^{-1}(t)=\int_{0}^{t} \psi^{2}(s) d s$ so

$$
t=\int_{0}^{A(t)} \psi^{2}(s) d s=\int_{0}^{t} \psi^{2}(A(s)) d A(s)=\int_{0}^{t} \psi^{2}(A(s)) \Phi^{2}(s) d s \quad \text { a.s. }
$$

so $\Phi^{2}(s)>0$ a.e. $d t \times d P$.

Case 2. If $E\left[A^{-1}(t)\right]=\infty$ for some $t$, we still have $M(t)=\int_{0}^{t} \Phi(s) d X(s)$ and $A(t)=\int_{0}^{t} \Phi^{2}(s) d s$. Assume $\Phi(s)=0$ on a $d t \times d P$ set of positive measure. Then $\Phi(s)=0$ for $0 \leqq s \leqq t_{0}$ on a set of positive $d t \times d P$ measure for some $t_{0}>0$. Fix such a $t_{0}$. Define

$$
\begin{aligned}
M^{\prime}(t) & =M(t) & & \text { if } 0 \leqq t \leqq t_{0} \\
& =M\left(t_{0}\right)+X(t)-X\left(t_{0}\right) & & \text { if } t>t_{0} .
\end{aligned}
$$


$M^{\prime}(t)$ is still a martingale adapted to $\mathscr{G}(t)$ and

$$
\begin{aligned}
\left\langle M^{\prime}\right\rangle_{t} & =A(t) & & \text { if } t \leqq t_{0}, \\
& =A\left(t_{0}\right)+t-t_{0} & & \text { if } t>t_{0} .
\end{aligned}
$$

Now $M^{\prime}(t)$ has the properties of Case 1 . That is $E\left[\left\langle M^{\prime}\right\rangle_{t}^{-1}\right] \leqq t+t_{0}$ for all $t \geqq 0$. However, $M(t)=M^{\prime}(t)$ for $0 \leqq t \leqq t_{0}$ so $M^{\prime}(t)=\int_{0}^{t} \Phi(s) d X(s)=M(t)$ for $t<t_{0}$, but using $M^{\prime}(t)$ we know $\Phi(s) \neq 0$ a.e. $d t \times d P$. This contradiction says $\Phi(s) \neq 0$ a.e. $d t \times d P$.

REMARK 1. If the converse to Theorem 1 were true, Theorem 2 would hold for $M(t) \in \mathscr{M}_{2}$.

REMARK 2. The above proof shows that if $\sigma\{X(s): s \leqq t\}=\sigma\{M(s): s \leqq t\}$ for $M(t) \in \mathscr{M}_{2}^{*}$, then both $\langle M\rangle_{t}$ and $\langle M\rangle_{t}^{-1}$ are absolutely continuous with respect to Lebesgue measure.

As an application of this theorem consider the following question discussed previously in [6]. Let $N(t)=\int_{0}^{t} \Phi(s) d M(s)$ where $M(s)=$ $X(A(s))$ belongs to $\mathscr{M}_{2}$. It is shown by example that one may not have $\lim _{\Delta t \rightarrow 0}(\Delta N(t) / \Delta M(t))=\Phi(t)$ for $M(t) \in \mathscr{M}_{2}$ [7]. However, if $\langle M\rangle_{t}$ has a positive derivative a.e. $d t \times d P$ so $M(t)=\int_{0}^{t} \alpha(s) d X(s)$ with $\alpha(s) \neq 0$ a.e. $d t \times d P$, then the convergence of the difference quotient to the integrand will hold [6]. The above theorem shows that $M(t) \in \mathscr{M}_{2}^{*}$ with $\sigma\{M(s): s \leqq t\}=$ $\sigma\{X(s): s \leqq t\}$ is also sufficient for this convergence.

The generalization of Theorem 2 to the case where one starts with two martingales $M(t)$ and $N(t)$ seems more difficult. Strong use was made of the fact that $X(t)$ was a Brownian motion, both in Lemma 2 and in the definition of $\mathscr{M}_{2}^{*}$. However, some analogue of Theorem 2 should hold for $M(t)$ and $N(t)$ belonging to $\mathscr{M}_{2}$ or $\mathscr{M}_{2}^{*}$.

4. Acknowledgement. The author wishes to thank the referee for his comments on this paper.

\section{BIBLIOGRAPHY}

1. R. M. Blumenthal and R. K. Getoor, Markov processes and potential theory, Pure and Appl. Math., vol. 29, Academic Press, New York, 1968. MR 41 \#9348.

2. K. È. Dambis, On decomposition of continuous submartingales, Teor. Verojatnost. i Primenen. 10 (1965), 438-448=Theor. Probability Appl. 10 (1965), 401-410. MR 34 \#2052.

3. J. L. Doob, Stochastic processes, Wiley, New York; Chapman and Hall, London, 1953. MR 15, 445.

4. L. E. Dubins and G. Schwarz, On continuous martingales, Proc. Nat. Acad. Sci. U.S.A. 53 (1965), 913-916. MR 31 \#2756.

5. D. L. Fisk, Sample quadratic variation of sample continuous, second order martingales, Z. Wahrscheinlichkeitstheorie und Verw. Gebiete 6 (1966), 273-278. MR 35 \#1078. 
6. D. Isaacson, Stochastic integrals and derivatives, Ann. Math. Statist. 40 (1969), 1610-1616. MR 40 \#5038.

7. - Continuous martingales with discontinuous marginal distributions, Ann. Math. Statist. 42 (1971), 2139-2142.

8. P. A. Meyer, Sur un théorème de Deny, Séminaire de Probabilités (Univ. Strasbourg, 1966/67), vol. I, Springer, Berlin, 1967, pp. 163-165. MR 38 \#2332.

9. —_, Probability and potentials, Blaisdell, Waltham, Mass., 1966. MR 34 \#5119.

Department of Mathematics and Department of Statistics, Iowa State UniVERSITY, AMES, IOWA 50010 